\title{
LICHENOID LESION AND ORAL SQUAMOUS CELL CARCINOMA OF THE TONGUE
}

H.B. Yaacob, W.C. Ngeow, P.L. Tan, A.R. Zainal Ariff. Lichenoid lesion and oral squamous cell carcinoma of the tongue. Annal Dent Univ Malaya 2002; 9: 23-26.

\begin{abstract}
The controversy on malignant transformation of oral lichen planus has always intrigued researchers. We present a case of lichenoid lesion and squamous cell carcinoma of the tongue, occurring in a middle aged Indian lady. The diagnosis and timing of these lesions is discussed.
\end{abstract}

Key words: malignancy, squamous cell carcinoma, lichenoid lesion.

\section{INTRODUCTION}

It is well known that the risk of malignant transformation of oral lichen planus (OLP) remains a controversial issue (1-9). Krutchkoff et al., who in 1978 critically reviewed 223 cases published between 1950 and 1976 found only 15 cases of malignant transformation of OLP, acceptable based on certain strict guidelines that they outlined (1). Their review revealed several shortcomings in the study of malignant transformation of OLP. Many reports lacked full historical data concerning previous carcinogen exposure whereas some described malignancy in oral locations anatomically remote from the OLP lesions. There were also several conspicuous and critical inadequacies identified with respect to the original diagnoses of OLP. Some initial diagnoses were made without a biopsy and therefore lacked histopathologic confirmation of what were clinically diagnosed as OLP. Other photomicrograph showed dubious gross features that give an impression of leukoplakia rather than OLP (1).

The more recent critical meta-analysis-type review of the literature from 1977 to 1999 by van der Meij et al., (2) which covered 28 studies and examined for the histologic documentation regarding malignant transformation of OLP, confirmed transformation in 10 of the reports (34\% of 98 reported cases). However they too, found that documentation deficiencies similar to those identified by Krutchkoff's group weakened the credibility of many follow-up studies and case reports published since that time.

Moreover, the presence of OLP and squamous cell carcinoma (SCC) are not rare, with some patients having both problems simultaneously (6) and the two processes may be unrelated to each other. Conversely,
Case Report

H.B. Yaacob ${ }^{1}$, W.C. Ngeow, ${ }^{2}$ P.L. $\operatorname{Tan}^{3}$ and

A.R. Zainal Ariff

${ }^{I}$ Professor

${ }^{2}$ Lecturer

${ }^{3}$ Former Dental Student

${ }^{4}$ Associate Professor

${ }^{2}$ Faculty of Dentistry, University of Malaya, 50603 Kuala Lumpur, Malaysia

Tel: 03 - 7967 4882. Fax: 03 - 79674534

E-mail: ngeowy@um.edu.my

Corresponding author - Dr. Ngeow Wei Cheong

some reports showed that atrophic and, or erosive LP has a higher risk of developing into cancerous lesions.

We report a case of lichenoid lesion and oral squamous cell carcinoma in a middle-aged Indian lady.

\section{CASE REPORT}

A 47 year-old Indian lady was referred to the Dental Faculty of the University of Malaya in October 1994. She complained of having a loose upper right molar and pain over the right side of her tongue, suspected to be due to trauma from teeth. There was ulceration on the right side of her tongue for the past two weeks. Intra-oral examination revealed a swelling and ulceration on the right lateral side of tongue, which was tender or palpation. The symptomatic ulcerated area was oval in shape with a size of $10 \times 5 \mathrm{~mm}$. She also suffered from generalised chronic adult periodontitis.

The patient was subsequently reviewed five days later. Since the pain persisted and the swelling remained, she was referred to the Oral Medicine clinic for further management. Intra oral examination showed a hyperaemic area on the lower right latero-ventral surface of the tongue with an ulcerated area below it. A complaint of burning sensation when eating spicy food was noted. The patient did not admit to having quid chewing habit. No associated cutaneous lesion was found. An incisional biopsy of the lesion, performed under local anaesthesia in November 1994, yielded a histopathology result consistent with that of lichenoid lesion (Figure 1).

The patient failed to attend further follow-up. However, a private medical practitioner referred her back in August 1996 due to severe pain on the right side of the tongue. She complained that the ulcer on 


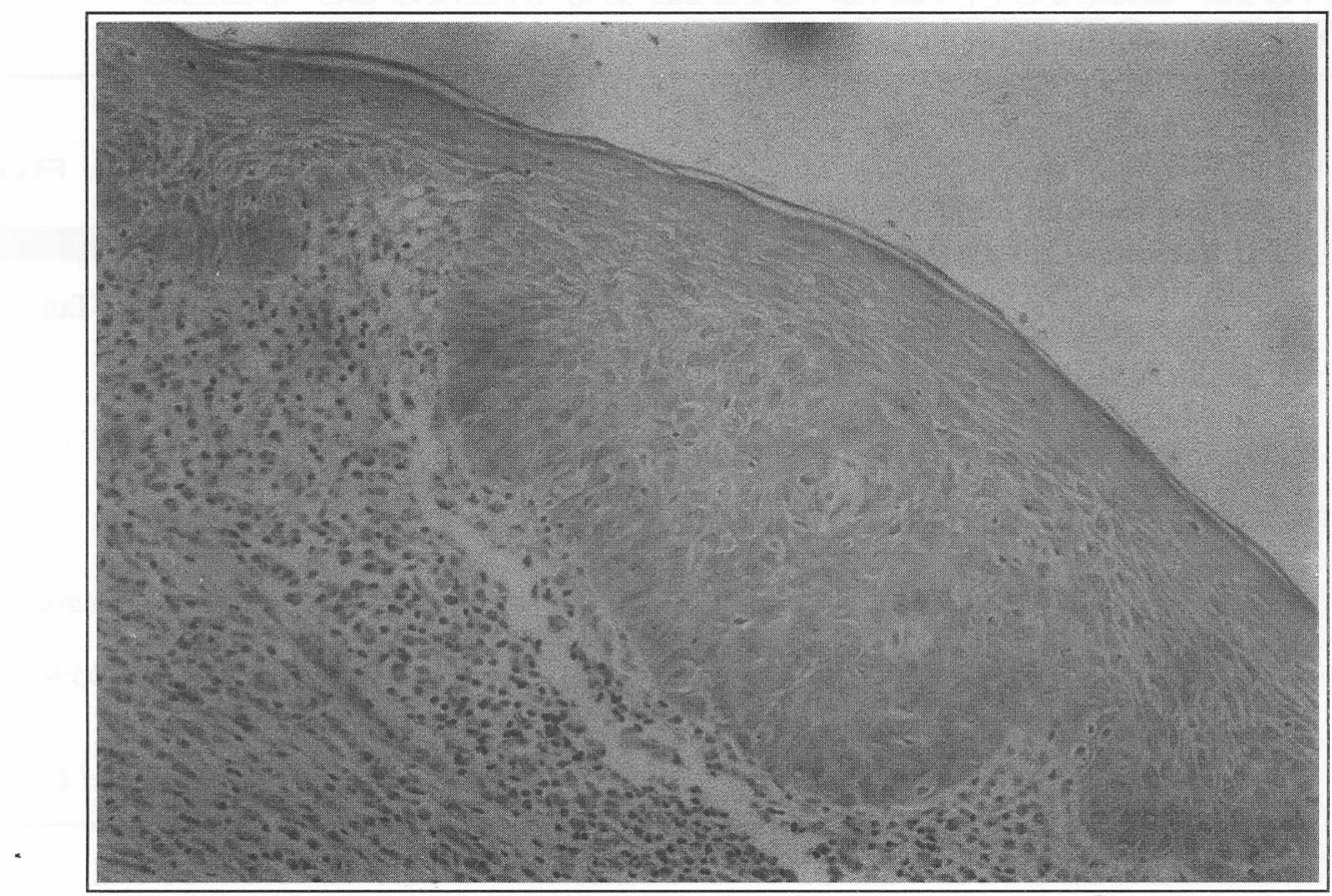

Figure 1: Photomicrograph of lichenoid reaction at the ventro-lateral border of the tongue.

The epithelium was intact but a band of chronic inflammatory cells was noted at the juxta-epithelial area.

(H \& E stain, X20)

the right side of tongue did not show any improvement for the past three years. An incisional biopsy was planned but again she did not keep her appointment.

In April 2000, she responded to an exercise of recalling OLP patients and returned to the Oral Medicine clinic complaining of pain and ulceration on the right ventral surface of tongue, which never healed since its first occurrence in 1994. Intra-oral examination revealed a hyperaemic area with ulceration, then resembling leukoplakia. On further questioning, she admitted to occasional tobacco use. An incisional biopsy, carried out under local anaesthesia, yielded a histopathology result consistent with that of a superficial invasive squamous cell carcinoma (Figure 2).

Excision of tumour was performed with the patient under general anaesthesia. Unfortunately, the surgical margin was not cleared even after two operations. It was then decided that hemiglossectomy of right side of the tongue be performed. The tongue was reconstructed with a radial forearm free flap. Healing was uneventful and the patient is currently free from recurrence.

\section{DISCUSSION}

The initial diagnosis of the patient concerned 7 years ago was lichenoid lesion on the right latero-ventral surface of her tongue. There was, in fact, hesitation to label the lesion typical OLP by the oral pathologist as the clinical presentation was not well described. The lesion was erythematous and ulcerated. In addition, there was no clinical photograph to confirm the appearance of erosive lichen planus. The patient claimed that the ulcer never healed. As noticed by Eisenberg and Krutchkoff (3), a problem that recurred in many cases of purported malignant transformation. of OLP is the strong suspicion that initial diagnostic errors may have been made. Diagnoses in such cases can be considered only presumptive at best. Even in instances where clinical photographs or photomicrographs were available, such evidence not only fails to corroborate the diagnosis of OLP, but instead suggested different entities (4-6). We find similar problem in diagnosing this case, as there was no earlier clinical photograph and detailed information regarding the appearance of the lesion. This explains the reason the histopathological report being consistent with lichenoid lesion instead of the typical label of OLP.

The patient recently admitted to occasional chewing of tobacco though initially denied doing so 7 years ago. Based on one of Krutchkoff's criteria, that is, tobacco habits be properly documented to help distinguished between true malignant transformation and conventional carcinomas present in the oral cavity of OLP patients, this case suggests that malignancy may have occurred simultaneously with the lichenoid lesion (6) instead of frank malignant transformation. Without a clear description of the original lesion clinically, i.e. presence of whitish striae and/or white plaques, the terminology of lichenoid lesion remains a histological terminology in this case. Interestingly, this histopathological picture has also been described in mucosal changes due to tobacco chewing/quid 


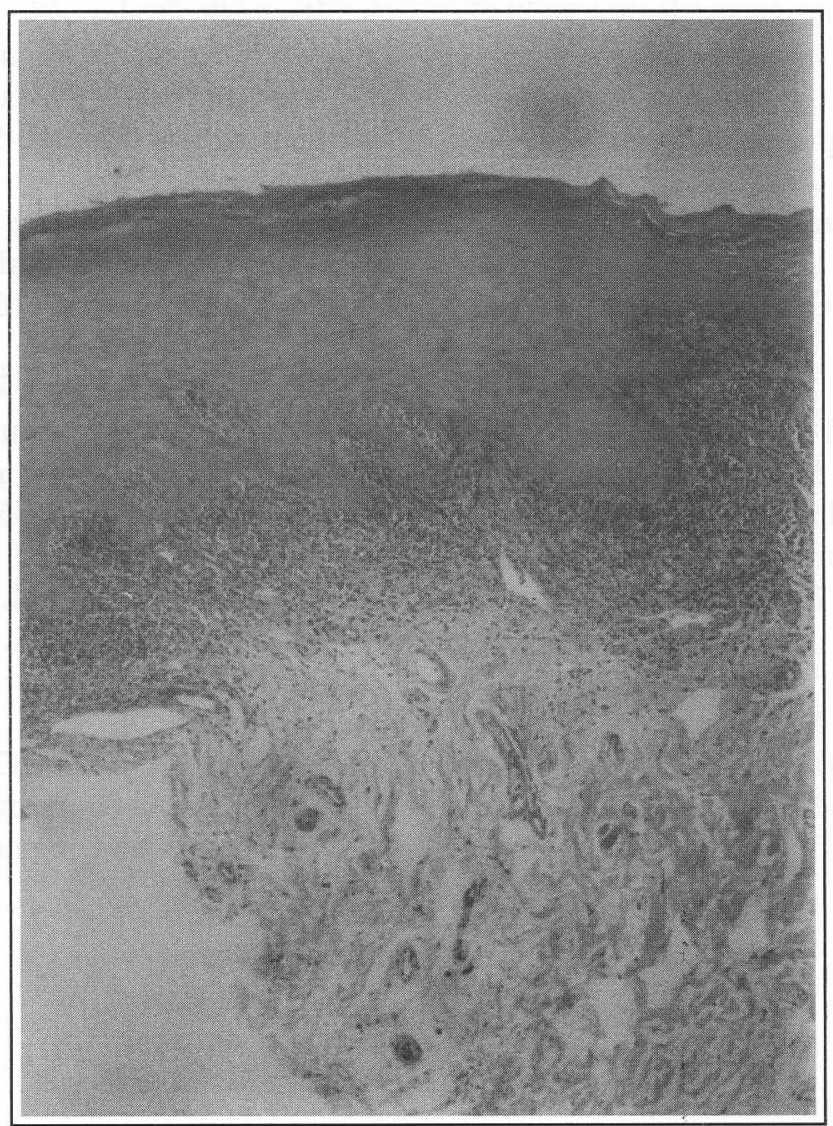

Figure 2: Photomicrograph shows dyplastic feature and superficial invasion by the underlying connective tissue by malignant epithelial cells.

(H\&E x 20)

chewing regardless of the clinical diagnosis of lichen planus, betel-quid lichenoid lesion, chewer's mucosa and leukoplakia. In fact such histological descriptions seen in lichenoid lesion have also been seen in margins of oral cancer where histologically there was dysplasia. Based on available data in the patient's record, the SCC would probably have arisen from damaged mucosa/ulcerative lichenoid lesion but was not picked up during the first biopsy as the specimen may not be representative.

As suggested by Eisenberg and Krutchkoff (3), it would be improper to consider such malignant conditions as the natural progression of an innately premalignant process. More accurately, they suggested that such instances be considered as conventional oral cancers that develop through exposure to known carcinogens in patients. They also suggested that any erosive or atrophic lesion would predisposed to accentuated influence of recognised carcinogens, which renders it more susceptible to malignant degeneration. We believe this case is an example of those suggested by Eisenberg and Krutchkoff.

It has been suggested that the erosive or atrophic forms of OLP have the greater potential to undergo malignant transformation compared to other variants of LP $(5,7)$. Many investigators favoured the concept that the atrophic or erosive forms of OLP predispose the mucosa to damage from other carcinogenic agents. According to this view, the lesions themselves are not considered premalignant in the sense that erythroplakias of the oral cavity are $(6,8,9)$. The lesion presented in our patient was an ulcerative lesion located at the ventral surface of the tongue for the duration of 7 years. Thus, whether lichen planus was present or not, such an ulcerative presentation itself may favoured the concept that such lesion predispose the mucosa to damage from carcinogenic agents.

\section{CONCLUSION}

More attention should be given in the case of atrophic or erosive lesion, especially on the ventral surface of the tongue and the floor of the mouth due to the high risk of malignancy in these areas. Any white, red or ulcerative lesion found in these areas is perhaps more appropriately biopsied with a wide margin in order to ensure a representative specimen is obtained.

\section{REFERENCES}

1. Krutchkoff DJ, Cutler LS, Laskowski S. Oral lichen planus: The evidence regarding potential 
malignant transformation. J Oral Pathol 1978; 7: 1-7.

2. van der Meij EH, Schepman KP, Smeele LE, van der Wal JE, Bezemer PD, van der Waal I. A review of the recent literature regarding malignant transformation of oral lichen planus. Oral Surg Oral Med Oral Pathol Oral Radiol Endod 1999; 88: 307-10.

3. Eisenberg E, Krutchkoff DJ. Controversies in oral pathology: Lichenoid lesions of oral mucosa. diagnostic criteria and their importance in the alleged relationship to oral cancer. Oral Surg Oral Med Oral Pathol 1992; 73: 699-704.

4. Murti PR, Bhonsle RB, Daftary DK, Gupta PC, Mehta FS, Pindborg JJ. Malignant potential of oral lichen planus: observation in 722 patients from India. J Oral Pathol Med 1986; 15: 71-7.
5. Katz RW, Brahim JS, Travis WD. Oral squamous cell carcinoma arising in a patient with longstanding lichen planus. Oral Surg Oral Med Oral Pathol 1990; 70: 282-5.

6. Lind PO, Koppang HS, Aas E. Malignant transformation in oral lichen planus. Int J Oral Surg 1985; 14: 509-16.

7. Marder MZ, Deesen KC. Transformation of oral lichen planus to squamous cell carcinoma: a literature review and report of case. J Ann Dent Assoc 1982; 105: 55-60.

8. Kaplan K, Barnes L. Oral lichen planus and squamous carcinoma. Arch Otolaryngol. 1985; 111:543-7.

9. Barnard NA, Scully C, Eveson JW. Cunningham S, Porter SA. Oral cancer development in patients with oral lichen planus. J Oral Pathol Med 1993; 22: 421-4. 